\title{
Combination of methylprednisolone pulse therapy and remission inducing drugs in rheumatoid arthritis
}

\author{
TROELS MØRK HANSEN, EBBE DICKMEISS, HENNING JANS, \\ THORSTEN INGEMANN HANSEN, MARGRETHE INGEMAN-NIELSEN,
} AND IB LORENZEN

From the Department of Rheumatology, the Blood Bank, and the Department of Radiology, Hvidovreo Hospital, University of Copenhagen and Kong Christian X'th Gigthospital, Graasten, Denmark

SUMMARY Thirty patients with rheumatoid arthritis were allocated to either methylprednisolon $\vec{k}$ pulse therapy or placebo at the beginning of treatment with either gold salts, penicillamine, of azathioprine. Methylprednisolone pulse therapy produced an immediate but temporars anti-inflammatory effect lasting for a maximum of four to eight weeks. It also caused a lasting. depression of serum IgG, but no effect was observed on the proportion of $\mathrm{T}$ and $\mathrm{B}$ lymphocytes proliferative responses, or on concanavalin A induced suppressor cell activity, and there was n节. effect on the amount of circulating immune complexes. The bone mineral content decrease $ळ$ similarly in the two groups, and methylprednisolone pulses had no effect on the progression of erosions on $x$ rays during an observation period of eight months. A single pulse of methylprednisolone can give a short lasting anti-inflammatory effect but is of little or no value if the long term treatment of rheumatoid arthritis.

Key words: glucocorticoids, gold salts, penicillamine, azathioprine, $x$ rays, bone mineral content禺 immunoglobulins, T lymphocytes, B lymphocytes.

Pulse therapy with $1 \mathrm{~g}$ methylprednisolone (MP) given intravenously to patients with rheumatoid arthritis (RA) can reduce the clinical signs of inflammation. $^{1-6}$ The improvement may last for several weeks, though MP is metabolised and excreted within a few days. ${ }^{7}$ It is thus possible that pulse therapy with MP may induce a long lasting anti-inflammatory effect with fewer side effects than continuous treatment with oral glucocorticoids.

The purpose of the present investigation was to study if treatment with $1 \mathrm{~g}$ MP given intravenously daily for three days at the beginning of treatment with a remission inducing drug (gold salts, penicillamine, or azathioprine) would improve the clinical response during an eight month observation period. The patients were examined regularly for clinical and laboratory signs of disease activity and for side effects. Furthermore, immunological variables were measured and $x$ rays of hands and feet were taken before treatment and after eight months.

Accepted for publication 22 September 1986

Correspondence to $\mathrm{Dr}$ Troels Mørk Hansen, Department of Rheumatology, Kong Christian X'th Gigthospital, DK-6300 Graasten, Denmark.

\section{Patients and methods}

The study was designed as a double blind trial with open therapeutic control.

\section{PAT IEN TS}

Thirty patients were entered with a median age of 6 years, range 25-79 years. All had classical of definite RA according to the criteria of the Amer ican Rheumatism Association. Informed conse according to the Helsinki II declaration was obtained. Patients who had had the disease for les than one year and patients who belonged to funco tional class IV were excluded. None of the patien had received treatment with remission inducing drugs (gold salts, penicillamine, levamisole, ant malarials, cytostatics) within the last month. $P\left(\frac{1}{1}\right.$ tients receiving oral glucocorticoids were included the dose could remain unchanged during the eight months of observation. All the patients fulfilled $a$ minimum of three of the following four criteria of disease activity: (a) erythrocyte sedimentation rato $($ ESR $)>28 \mathrm{~mm} / \mathrm{h} ;(b)$ duration of morning stiffnes $\geqslant 45 \mathrm{~min} ;(c)$ number of tender joints $\geqslant 6$; ( number of swollen joints $\geqslant 3$. 
Table 1 Description and stratification of the patients

\begin{tabular}{|c|c|c|}
\hline & $\begin{array}{l}\text { Methyl- } \\
\text { prednisolone }\end{array}$ & Placebo \\
\hline $\operatorname{Sex}(\mathbf{M} / \mathbf{F})$ & $5 / 10$ & $6 / 9$ \\
\hline Age $(>50 /<50)$ & $12 / 3$ & $12 / 3$ \\
\hline \multicolumn{3}{|l|}{ Duration of RA } \\
\hline ( $>2$ years $/<2$ years $)$ & $14 / 1$ & $11 / 4$ \\
\hline Functional class (I-II/III) & $13 / 2$ & $15 / 0$ \\
\hline SCAT $^{*}$ titre $(\geqslant 40 /<40)$ & $11 / 4$ & $12 / 3$ \\
\hline Erosions on $x$ ray $(+l-)$ & $14 / 1$ & $13 / 2$ \\
\hline $\begin{array}{l}\text { Glucocorticoids orally } \\
(+/-)\end{array}$ & $1 / 14$ & $2 / 13$ \\
\hline $\begin{array}{l}\text { Type of treatment started } \\
\text { after pulse therapy (gold } \\
\text { salts/penicillamine/ } \\
\text { azathioprine) }\end{array}$ & $7 / 5 / 3$ & $7 / 2 / 6$ \\
\hline
\end{tabular}

${ }^{*}$ SCAT $=$ sheep cell agglutination titre.

Further details on the patients and their stratification are given in Table 1.

\section{T REA T M E N T}

The principle of minimisation ${ }^{8}$ was used for allocation of the patients to either pulse therapy with MP or saline. Fifteen patients were given $1 \mathrm{~g} \mathrm{MP}$ intravenously in $500 \mathrm{ml}$ physiological saline daily for three days (days $0,1,2$ ), while the other 15 patients were given $500 \mathrm{ml}$ physiological saline only. Immediately after the pulse therapy all the patients were started on either gold salts, penicillamine, or azathioprine. The patients started on gold salts received $50 \mathrm{mg}$ weekly unless they had experienced side effects or no response in a previous course of gold treatment. If so they were given penicillamine, starting with $150 \mathrm{mg}$ daily and increasing the dose every three weeks by $150 \mathrm{mg}$ to a maximum of $450-600 \mathrm{mg}$. Patients who had had side effects or no clinical response in an earlier treatment with penicillamine were given azathioprine $100-150 \mathrm{mg} /$ day.

\section{CLINICAL INVESTIGATIONS}

The patients were examined by the same 'blind' observer before the pulse therapy and after 1-2 weeks, 4-8 weeks, $12-16$ weeks, and 32 weeks. The number of tender joints and the number of swollen joints were recorded. The patients' assessment of pain on a $100 \mathrm{~mm}$ visual analogue scale and the duration of morning stiffness were registered.

\section{LABORATORY INVESTIGATIONS}

The following variables were measured before the pulse therapy and at days 3, 7, and 14: ESR, haemoglobin $(\mathrm{Hb})$, number of granulocytes, lymphocytes and platelets in the blood, serum iron, potassium, sodium, cholesterol, triglyceride, creatinine, transaminase, and fasting blood glucose. ESR, $\mathrm{Hb}$, granulocytes, and platelets were determined monthly thereafter. Before treatment and after 1 , 16 , and 32 weeks serum calcium, phosphate, and alkaline phosphatase were measured. Serum albumin, IgG, IgA, IgM, and sheep cell agglutination titre (SCAT) were measured before and after 14 days, four months, and eight months. An adrenal stimulation test with Synacthen (Ciba) was performed before and 14 days after the pulse therapy. Circulating immune complexes were sought in fresh frozen $\left(-80^{\circ} \mathrm{C}\right)$ serum samples before the pulse therapy and after one week and four months, by means of a complement consumption test and a polyethyleneglycol precipitation test as previously described. ${ }^{9}$

Subgroups of lymphocytes were measured before the pulse therapy and after one week and four months using the $E$ rosette technique and biotin conjugated anti-Leu-2a and anti-Leu-3a (BectonDickinson). The proliferative response to mitogens (phytohaemagglutinin concanavalin $\mathrm{A}$, and pokeweed mitogen) and the activity of concanavalin $A$ induced suppressor cells on the proliferative response of autologous lymphocytes to pokeweed mitogen were also determined. The details of the methods have previously been published. ${ }^{10}$

\section{RAYS}

Hands, wrists, and feet were examined radiographically before the pulse therapy and after eight months. In each patient 46 joints were examined by two doctors together. The presence of erosions of at least $1 \mathrm{~mm}$ depth and any increase in the erosions after eight months were recorded.

BONE MINERAL CONTENT

Forearm bone mineral content was measured before treatment and after four and eight months by osteodensitometry with ${ }^{125} \mathrm{I}$ as the radiation source.

STATISTICAL EVALUATION

A Mann-Whitney rank sum test was used for a comparison of the MP treated group with the placebo group. Only $p$ values less than 0.05 were regarded as significant.

\section{Results}

MP treatment caused an immediate decrease in the signs of inflammation. There was a marked fall in ESR on days 3 and 7, but by day 14 the ESR had increased to pretreatment values and did not differ from the placebo group during the rest of the observation period (Fig. 1a). The number of tender and swollen joints and the patients' estimate of pain and morning stiffness were decreased one to two weeks after the MP pulse therapy, but no differ- 
(a)
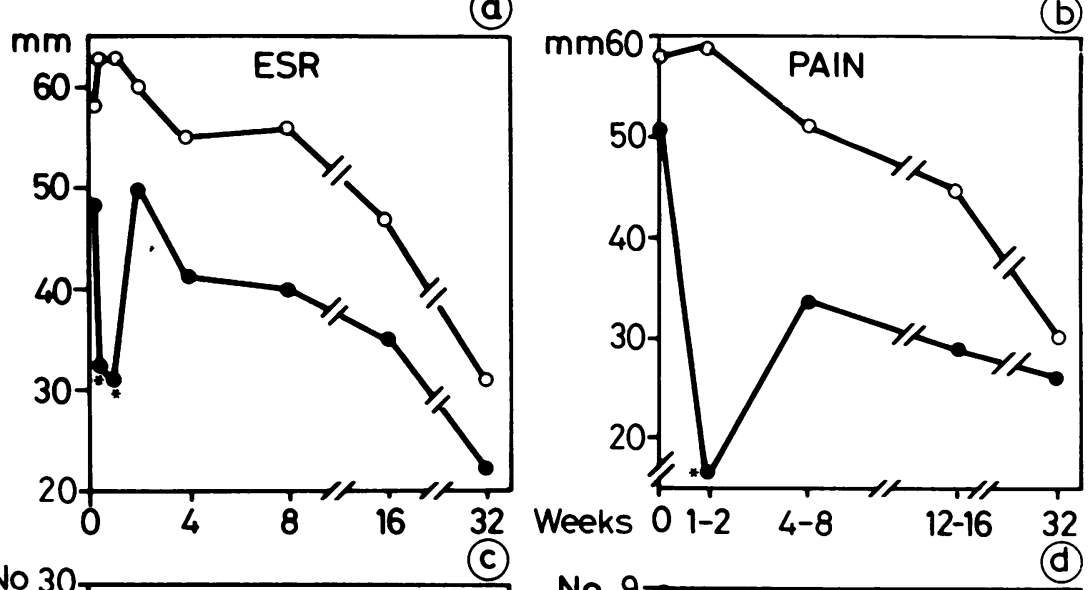

No 30

(c)

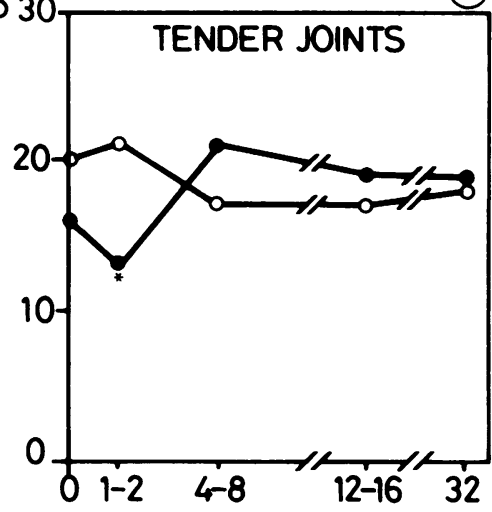

No

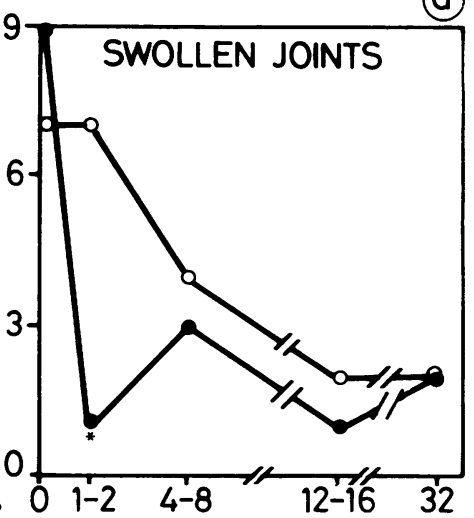

(e)
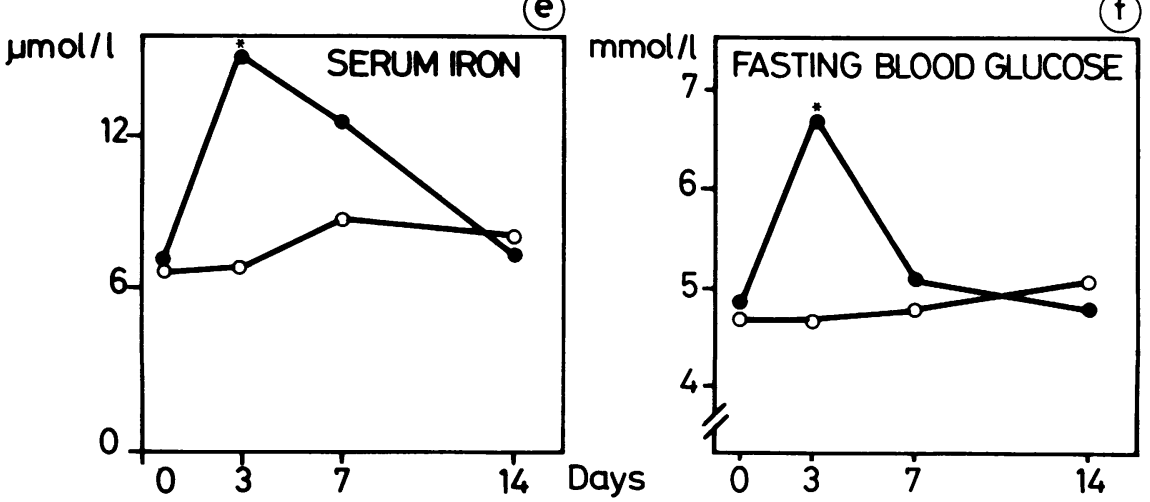

ences were detected between the MP and placebo treated patients after four to eight weeks or later (Figs 1b-d). MP treatment caused an increase in blood glucose and in serum iron on day 3 with normalisation on day 7 (Figs $1 \mathrm{e}$ and $\mathrm{f}$ ). $\mathrm{Hb}$ was increased on day 7 after MP treatment (Fig. 1g), and the blood granulocytes increased on days 3 and 7 and showed a slight increase at week 8 (Fig. 1h after MP treatment. No effect of MP was observea on the blood lymphocytes or platelets. Serum Ig $\mathbb{Q}$ and IgA were decreased two weeks after MP treatment, and the decrease in IgG was also 


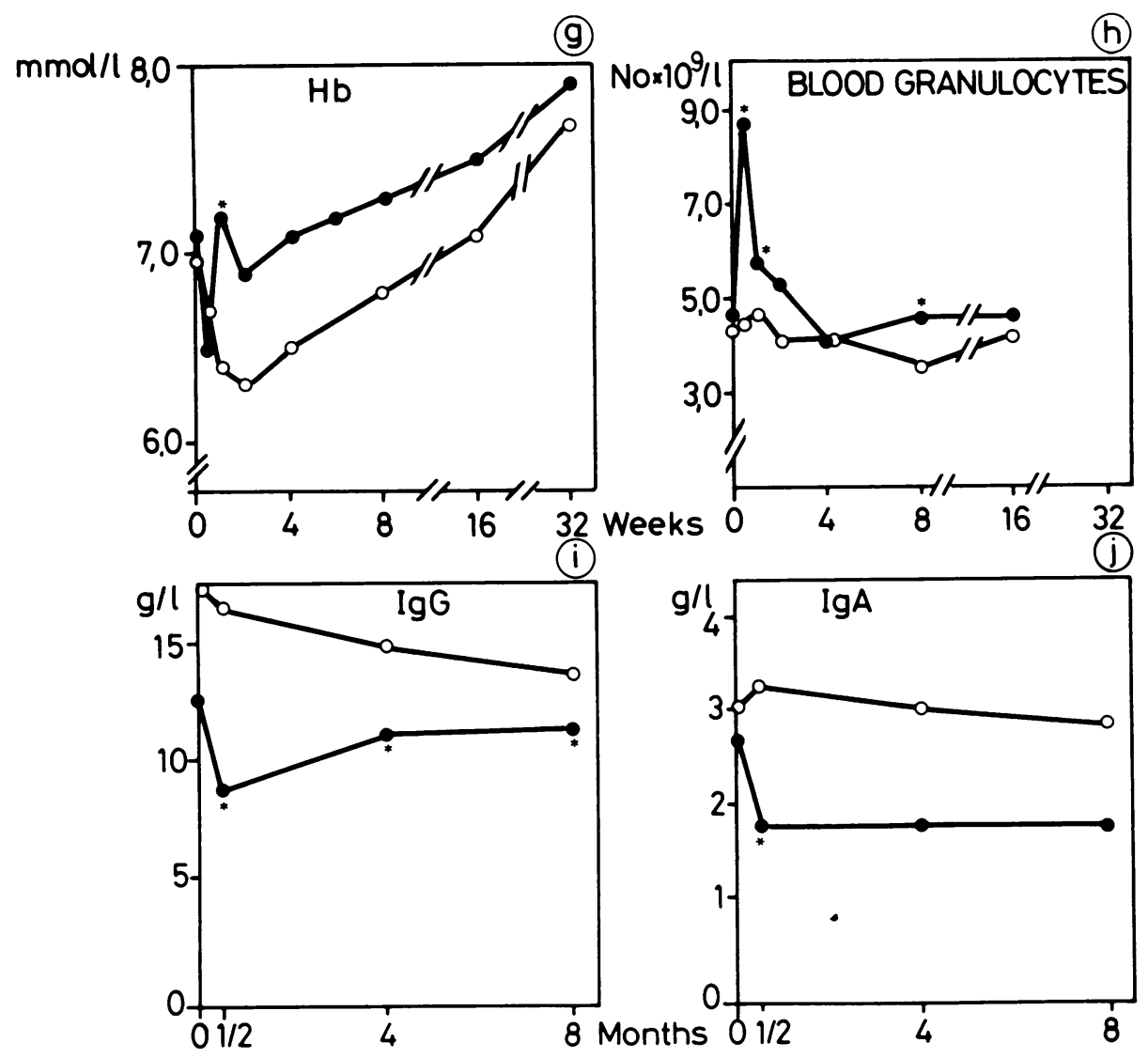

Fig. 1 The effect of $1 \mathrm{~g}$ methylprednisolone pulses given intravenously on three consecutive days. (a) ESR; (b) pain; (c) tender joints; (d) swollen joints; (e) serum iron; ( $f$ ) fasting blood glucose; $(g) \mathrm{Hb}$; (h) blood granulocytes; (i) IgG; (j) $I g A . \longrightarrow=$ methylprednisolone treated group, $\bigcirc-\mathrm{O}-$ placebo treated group. ${ }^{*} p<0 \cdot 05$.

significant after four and eight months (Figs $1 \mathrm{i}$ and j). There was no effect on serum IgM. MP treatment had no detectable effect on serum potassium, sodium, calcium, phosphate, transaminase, alkaline phosphatase, cholesterol, triglyceride, creatinine, albumin, SCAT, proportions of $\mathrm{T}$ and $\mathrm{B}$ lymphocytes, proliferative response to concanavalin $\mathrm{A}$, phytohaemagglutinin, and pokeweed mitogen, or on the concanavalin A induced suppressor cell activity. Circulating immune complexes were detected in six MP treated and in five placebo treated patients. No effect of the MP treatment was observed.

The adrenal stimulation test was normal in all patients 14 days after the MP therapy.

Radiographic examination showed progression of erosions during the eight months in nine patients, four MP treated and five placebo treated. In three patients in each group new erosions were detected.
Bone mineral content decreased by $2.5 \%$ (median) (range $-6.4 \%$ to $13.6 \%$ ) in the MP treated group and by $3.9 \%$ (median) (range $-8.1 \%$ to $\mathbf{9 . 3 \%}$ ) in the placebo treated group. There was no statistically significant difference between the two groups.

\section{Discussion}

Pulse therapy with $1 \mathrm{~g}$ MP on three consecutive days caused an immediate decrease in inflammatory symptoms in patients with RA. The fall in ESR only lasted for one week, and the effect on clinical signs of joint inflammation and the patients' own estimate of pain relief and decrease in morning stiffness had disappeared four to eight weeks after the pulse therapy. This is a slightly shorter duration than reported by others ${ }^{2-6}$ but similar to the observation 
by Gottlieb et al. ${ }^{1}$ In most studies single pulses of $1 \mathrm{~g}$ MP have been used. The use of $3 \mathrm{~g}$ MP given as $1 \mathrm{~g}$ daily on three consecutive days does not seem to be an advantage over a single dose of $1 \mathrm{~g} \mathrm{MP}$.

All patients were started on a remission inducing drug (gold salts, penicillamine, or azathioprine) at the time of the pulse therapy. There was an improvement in both the MP treated and placebo pulse treated patients during eight months of observation, but apart from a decrease in serum IgG no effect of MP on clinical or laboratory variables lasted for more than one month. Also the progression of erosions as shown by $x$ rays was similar in the two groups. Neumann et al have suggested that the use of a MP pulse might accelerate the response to remission inducing drugs, ${ }^{6}$ but their conclusion might be erroneous owing to their comparison with historical data.

The immediate increase in serum iron after MP treatment is probably secondary to the inhibition of inflammation as the inflammatory reaction causes a decrease in serum iron with a deposition of iron in the reticuloendothelial system. The temporary decrease in inflammation may explain the slight increase in $\mathrm{Hb}$ on day 7 in MP treated patients. The increase in blood glucose after MP treatment can be a problem in diabetic patients, but overt diabetes was not observed in our patients.

The well recognised increase in blood granulocytes during therapy with glucocorticoids also lasted less than 14 days. A number of effects of glucocorticoid pulse therapy have been reported on blood leucocytes during the first few hours. The adherence of granulocytes diminishes, ${ }^{11}$ there is a fall in the number of monocytes and lymphocytes, ${ }^{12}$ in particular $\mathrm{T}$ lymphocytes. ${ }^{13}$ The responses to stimulation with concanavalin $A$ and pokeweed mitogen decrease. ${ }^{12}$ These changes have been claimed to be due to a redistribution of the mononuclear cells in the blood, and a normalisation occurs within 24 hours. We were unable to detect any effect on the lymphocyte proportions or functions one week after pulse therapy. Also we were not able to detect any qualitative effect on immune complexes, though a quantitative depression of immune complexes has been reported. ${ }^{3}$ The long lasting decrease in serum IgG has also been observed by others. ${ }^{3-5}$ This is in contrast with the short duration of the other effects of the MP. The reason for this is unexplained but does not relate to a prolonged decrease in the inflammatory activity.

MP is metabolised and excreted within a few days, ${ }^{7}$ and no adrenal depression is detectable 14 days after the pulse therapy. No life threatening side effects have been reported in patients with RA. Central nervous system manifestations with seiz- ures, hemiplegia, and psychosis have been reporteक़ after pulse therapy in patients with systemic lupus. erythematosus. ${ }^{14}{ }^{15}$ Sudden deaths within 24 hours? after pulse therapy have been reported in ren $\Phi$ allograft recipients in patients with systemic lupus. erythematosus with renal involvement and in pas tients with pericarditis. ${ }^{16-18}$ In some patients myocardial infarctions were detected and in other different arrythmias. MP pulse therapy may be safe. in patients with uncomplicated RA if the infusion is given slowly in a minimum of $30 \mathrm{~min}$. In RA patientड with cardiac symptoms or severe nephropathy the indication for MP pulse requires particularly carefu偻 consideration.

In conclusion, MP pulse as single therapy in R cannot be recommended. To sustain the inhibition of inflammatory activity MP pulses must be given with intervals of no longer than four weeks. The question of whether repeated pulses with MP during the first six months of treatment with a remissio inducing drug can affect the long term prognosis i RA is still unanswered. It also needs to be elucidated whether doses of MP smaller than $1 \mathrm{~g}$ are् effective and whether repeated doses of MP have fewer side effects than other types of glucocorticoio treatment.

\section{References}

1 Gottlieb N L, Riskin W, Vidal A, Hyer F, McKinney Methylprednisolone (MP) pulse therapy in rheumatoid arthritis Clin Res 1980; 28: 146a.

2 Williams I A, Baylis E M, English J. High dose intravenous methylprednisolone (pulse therapy) in the treatment of rheuma toid disease. Scand J Rheumatol 1981; 10: 153-5.

3 Liebling M R, Leib E, McLaughlin K, et al. Pulse methylpre nisolone in rheumatoid arthritis. Ann Intern Med 1981; 9 21-6.

4 Williams I A, Baylis E M, Shipley M E. A double-bling placebo-controlled trial of methylprednisolone pulse therapy active rheumatoid disease. Lancet 1982; i: 237-40.

5 Forster P J G, Grindulis K A, Neumann V, Hubball S McConkey B. High-dose intravenous methylprednisolone $\underline{m}$ rheumatoid arthritis. Ann Rheum Dis 1982; 41: 444-6.

6 Neumann V, Hopkins R, Dixon J, Watkins A, Bird H, Wrigd V. Combination therapy with pulsed methylprednisolone rheumatoid arthritis. Ann Rheum Dis 1985; 44: 747-51. N

7 Baylis E M, Williams I A, English J, Marks V, Chakraborty $\$ ? High dose intravenous methylprednisolone 'pulse' therapy in patients with rheumatoid disease. Eur J Clin Pharmacol 198\% 21: $385-8$.

8 Taves D R, Rochester N Y. Minimization: a new method 9 assigning patients to treatment and control groups. Clign Pharmacol Ther 1974; 15: 443-53.

9 Jans H, Dybkjaer E, Halberg P. Circulating immune complexक in healthy persons. Scand J Rheumatol 1982; 11: 194-8. +

10 Hansen T M, Dickmeiss E, Gerstoft J, Jans H, Halberg F Lorenzen I. Treatment of rheumatoid arthritis with bestatio. Clin Trials $J$ 1984; 21: 265-76.

11 Perez D, Kimberley R P, Kaplan H B, Edelson H, Inman R \&. Goldstein I M. Effect of high-dose methylprednisolone infusi@g on polymorphonuclear leukocyte function in patients with systemic lupus erythematosus. Arthritis Rheum 1981; 24: 641-4.

12 Fauci A S, Dale D C. The effect of in vivo hydrocortisone \&n 
subpopulations of human lymphocytes. J Clin Invest 1974; 53: 240-6.

13 Fan P T, Yu D T Y, Clements P J, Fowlston S, Eisman J, Bluestone $\mathbf{R}$. Effect of corticosteroids on the human immune response: comparison of one and three daily $1 \mathrm{gm}$ intravenous pulses of methylprednisolone. J Lab Clin Med 1978; 91: 625-34.

14 Suchman A L, Condemi J J, Leddy J P. Seizure after pulse therapy with methyl prednisolone. Arthritis Rheum 1983; 26: 117.

15 Ayoub W T, Torretti D, Harrington T M. Central nervous system manifestations after pulse therapy for systemic lupus erythematosus. Arthritis Rheum 1983; 26: 809-10.

16 Williams I A, Warren D J, Smith R S. High-dose prednisolone. Lancet 1983; i: 593-4.

17 Bocanegra T S, Castañeda M O, Espinoza L R, Vasey F B, Germain B F. Sudden death after methylprednisolone pulse therapy. Ann Intern Med 1981; 95: 122.

18 Moses R E, McCormick A, Nickey W. Fatal arrhythmia after pulse methylprednisolone therapy. Ann Intern Med 1981; 95: 782. 